\title{
Service and Network Management Interworking in Future Wireless Systems
}

\author{
V. Tountopoulos, V. Stavroulaki, P. Demestichas, N. Mitrou, and M. Theologou \\ National Technical University of Athens \\ Department of Electrical Engineering and Computer Science \\ Division of Communication, Electronic and Information Engineering, Telecommunications \\ Laboratory \\ 9 Heroon Polytechneiou Street, Zographou 15773, Athens, GREECE \\ Tel: + 30177214 95, Fax: + 3017722534 \\ vttounto@telecom.ntua.gr
}

\begin{abstract}
The need for seamless communications in future wireless networks imposes the development of distributed management systems, in which we can distinguish between different business entities, the Service and Network Providers. Such a highly dynamic environment enables the Service Providers to associate with multiple Network Providers and choose the best ones, according to user satisfaction and network's reward criteria. In this direction, this paper presents a Service Management System from the SP's perspective (SP-SMS). The SP-SMS can be separated into two planes, namely the service and network resource plane, and three layers, the Session Configuration Layer, the Local Planning Layer and the Global Planning Layer. Our study focuses on the Local Planning Layer and the role of the related components, which are introduced to describe the functionality of this layer. Through representative results, we demonstrate the superiority of the proposed model, in terms of utilisation and aggregate revenue.
\end{abstract}

\section{Introduction}

The liberalisation of telecommunications market and the success of future wireless systems require the provision of sophisticated and demanding services. The attainment of seamless communication imposes the adaptability of service requirements to the characteristics of the involved networks, without violating the limitations of physical infrastructure. The use of such services and applications usually requires a minimum Quality of Service (QoS), but the adoption of flexible resource allocation mechanisms comes up as an extremely challenging and interesting perspective.

It is obvious that a distributed, decentralised architecture for future wireless cellular systems is absolutely necessary to come up with stringent application requirements and dynamically varying available resources. The distinction between different entities in such systems enables the coupling of difficult radio infrastructure with the adaptability and scalability of multimedia service characteristics. In this direction, the introduction of Service Provider (SP) and Network Provider (NP) entities contributes to an efficient and aspiring approach to the problem. 
The scarcity of the radio resources and the versatility of the environment conditions, i.e. the time-variant traffic load, mobility levels and interference conditions, lead to degradations on the quality levels of the offered services. Thus, the competition between various SPs and NPs enables the effective exploitation of the cellular infrastructure, as well as the satisfaction of users' demands and requirements.

The scope of this paper is to present parts of the functional architecture of a Service Management System from the SP's perspective (SP-SMS), focusing on the functionality of the Local Planning Layer. Such a system enables the dynamic cooperation of an SP with the most appropriate NPs, in terms of user satisfaction and cost efficiency, in a competitive environment. More specifically, it addresses the problem of Service Configuration and Network Provider Selection (SCNPS), which will be introduced as part of the local planning layer components' logic.

The rest of the paper is organised as follows: Section 2 presents the general assumptions for a future wireless management system, regarding the policies of SPs and NPs. Section 3 focuses on the functionality at the local planning layer, giving a mathematical approach to the SCNPS problem. Section 4 presents a set of results for the evaluation of the proposed architecture. Finally, Section 5 includes concluding remarks.

\section{System Model}

This section presents briefly the objectives of the different entities (SPs and NPs) in a future wireless cellular system. It, also, gives the general structure of the proposed management system and describes the functionality of the involved layers.

\subsection{SP Objectives and Policies}

The SP role is to provide users with a set of services. Each SP has a volume of users, who have been subscribed to some or all the services available in this SP and have been sorted out into classes (user classes), according to their preferences in priority access to services. Each subscriber has been associated with a service usage profile, which models the behaviour exhibited by a typical subscriber of the user class, with respect to a specific service.

The users of each class have a set of permissible quality levels associated with each service, offered from the SP. Each quality level has been assigned a maximum tolerable price (tariff) and a utility measure. This factor is used to express the preference of a user class to some quality levels with respect to other permissible ones. It is assumed that the information, regarding permissible quality levels, maximum prices, utility levels, etc., is stored in the profiles of the different user classes.

Of the main objectives of an SP is to determine a reference quality level for a given service, which represents the minimum bandwidth required for the support of this service. During a session, the SP can allocate the service to a higher quality level, if this is acceptable. On the other hand, the SP is responsible for the accommodation of users to the most appropriate NP, according to quality and cost criteria, which can secure at least the reference quality level for the support of the selected service. 


\subsection{NP Policies}

The accomplishment of the SP role requires the co-operation with NPs. The NP role is to offer the network-level connectivity necessary for supporting the services. Within a portion of the service area, each NP can support up to a certain number of SP subscribers per quality level and imposes a tariff for the provision of a service at a quality level. The tariffs are determined by the NP policies, taking into account parameters, such as the managed network status, the volume of resources that the SP uses (either globally or in a specific area of the network), the area of the network, and the time zone.

\subsection{SP-SMS Design}

Without losing generality, we examine the SP-SMS management system from the perspective of a single SP. Figure 1 shows the general framework of a future wireless management system, depicting clearly the distinction between the different planes and layers, described below.

A future wireless management system can be classified into two planes, the service plane and the network resource plane. Both SPs and NPs are involved in the service plane. Through the functionality that is framed therein, the SP is enabled to find, in each service area portion and time zone, the target quality levels and the best NPs, for each service and user class. The SP-SMS covers the SP-related parts of the service plane.

The network resource plane involves only NPs. At this plane, the NPs manage the resources of the network infrastructure so as to meet the agreements having been established with SPs. This plane can rely on legacy network management systems. In this respect, the service plane functionality of the NPs can be seen as a necessary extension to legacy network management systems. The extension will enable the interworking with SPs and the promotion of the NP infrastructure.

As it can be seen from figure 1, the SP-SMS is structured into three layers, namely the Session Configuration Layer, the lower one at the SP-SMS hierarchy, the Local Planning Layer and the Global Planning Layer. Each layer contains a component type, which can be differentiated from the other component types of the other layers by the time scale and the service area portion, on which it operates. It should be noted, here, that the components of the SP-SMS co-operate with corresponding entities of the NP that fall within the service plane. In the rest of this subsection, we describe the functionality of the different components.

The SP Session Configuration Components (SP-SCCs) are enabled to handle with the incoming sessions. They are responsible for the monitoring of the network performance and the notification for the modification of the network configuration, when this is needed. Each SP-SCC controls a subset of the service area and is controlled by the components of the higher layers. The SP-SCC configuration specifies for each service and user class the target quality level that should be offered by the NPs, the best ones that can offer the target quality level and the maximum demand volume that each of them can accommodate. 


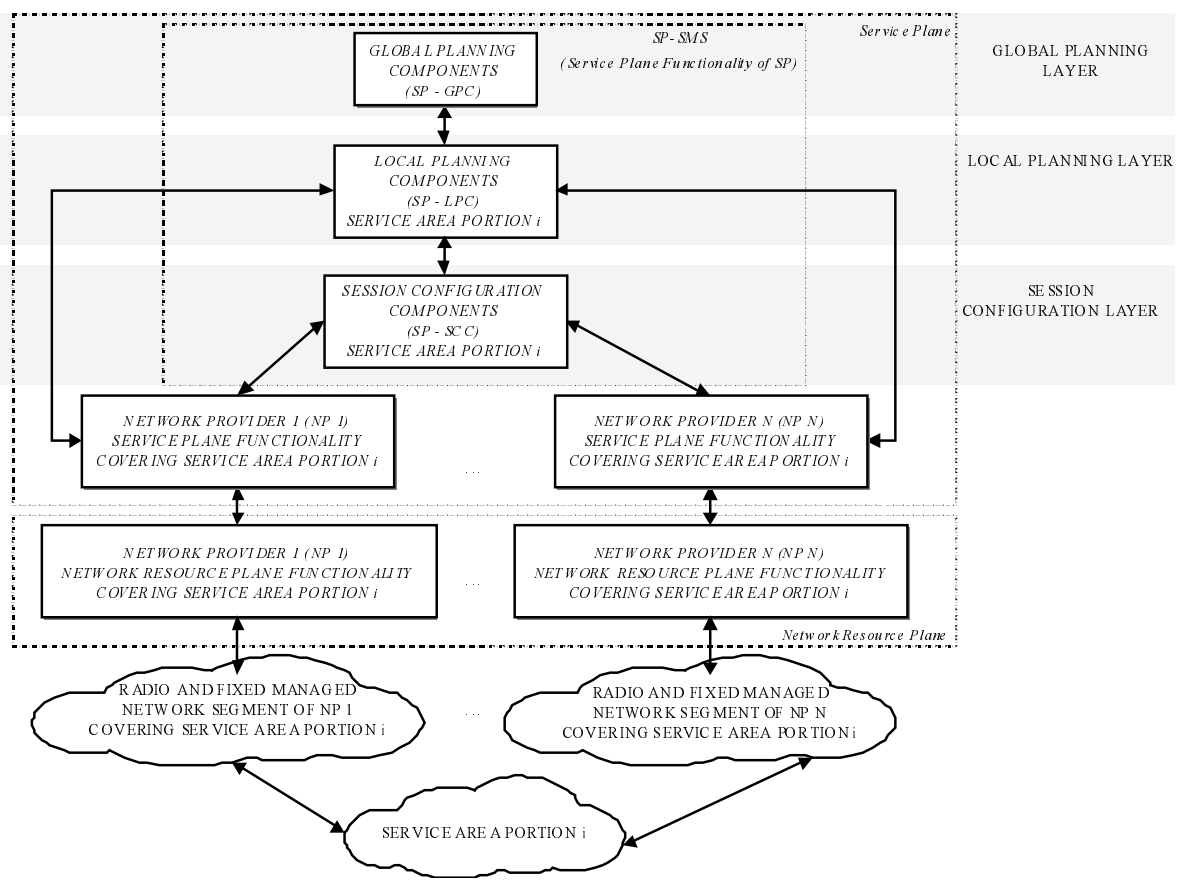

Fig. 1. Layers, components and high-level distribution pattern in the management framework

The SP Local Planning Components (SP-LPCs) control the counterpart portions of the service area as the SP-SCCs. They are responsible for the reconfiguration of the SP-SCCs, as it is described in section 3.

Finally, the SP Global Planning Components (SP-GPCs) focus over large subsets or the entire service area, and assist the components in the underlying layers in accomplishing their roles, by providing global policies and information.

\section{Local Planning Layer}

This section describes the functionality of the Local Planning Layer of the SP-SMS and the role of its components. More specifically, it addresses the problem of Service Configuration and Network Provider Selection (SCNPS), in order to clarify the concept of this layer in a future wireless management system. First, we present the possible structure of the management system, introducing the input parameters for our model, and then we give a mathematical presentation of the problem.

\subsection{High-Level Description of the SP-LPC Functionality (SCNPS Problem)}

Without loss of generality, we focus on a portion of the service area, denoted as $i$. As it has been stated above, there is an SP to control this area, which can be associated 
with a set of NPs, denoted as $N P_{\text {avail }}$. In service area $i$, we assume that the SP can offer a set $S$ of services to a set $U C_{s}$ of user classes, which have been subscribed to the service $s \in S$. We, also, define a set $Q_{s, u c}$ of permissible quality levels for the service $s$, which can be accessed by the subscribers of class $u c \in U C_{s}$. In the user's profile, it has been defined that the maximum acceptable price and the corresponding utility measure, when the service $s$ is offered to the users of class $u c$ at quality level $q\left(q \in Q_{s, u c}\right)$, are $\bar{c}_{s, u c, q}$ and $u_{s, u c, q}$, respectively.

We assume that each $n p \in \mathrm{NP}_{\text {avail }}$ can accommodate the specifications of the set of services $S$. There are two aspects that can configure the policy of an $n p$. First, the maximum network traffic load volume, $L_{\max }(n p)$, that the network provider can support in the service area portion $i$. Second, the set of tariffs, $c(n p, s, q)$, that the $n p$ will impose for the support of service $s$ at quality level $q\left(q \in \underset{u c \in U C_{s}}{\cup} Q_{s, u c}\right)$. These tariffs can depend on the network status, the resources used by the SP, the area of the network and the time zone.

Users that are found in service area portion $i$ send session requests to the controlling SP-SCC (SP-SCC- $i$ ). In response to the session request, the SP-SCC- $i$ indicates the target quality level $q$ and the best $n p$ for each service. The SP-SCC- $i$ reply is straightforward, based on the user information and on its configuration that has been done by the SP-LPC- $i$. The SP-SCC- $i$ monitors whether its configuration is appropriate for the service demand. In this respect, the SP-SCC- $i$ monitors the demand volume per service $s$ and user class $u c$, as well as the actual quality levels $Q_{s, u c}$ offered by the $N P_{\text {avail }}$, in the area $i$. In case the SP-SCC- $i$ identifies that its configuration is not appropriate for handling the demand for one or more services of the set $S$, it invokes the SP-LPC- $i$ and requests a modification. In response to the SP-SCC- $i$ invocation, the SP-LPC- $i$ must apply the SCNPS problem for modifying the SP-SCC- $i$ configuration.

The service demand pattern can be modelled through a vector $D_{s}=\{d(s, u c)$ $\left.\forall(s, u c) \in\left(S \times U C_{s}\right)\right\}$. Each element of the vector, $d(s, u c)$, corresponds to the demand for service $s$ that originates from the users of class $u c$ and should be accommodated by the SP, within the service area portion $i$ and time zone.

\subsection{Mathematical Formulation of the SCNPS Problem}

The SCNPS problem has two general objectives. First, to compute an allocation of the service demand pattern to quality levels, $A_{Q L}=\{q l(s, u c) \mid \forall(s, u c) \in$ 
$\left.\left(S \times U C_{s}\right)\right\}$. Each element of the allocation, $q l(s, u c)\left(q l(s, u c) \in Q_{s, u c}\right)$, is the target quality level, at which the users of class $u c$ should access service $s$ for the specific time zone and service area portion. The second objective of the SCNPS problem is to compute an allocation of the service demand pattern to network providers, $A_{N P}=\left\{r(n p, s, u c) \mid \forall(n p, s, u c) \in\left(N P_{\text {avail }} \times S \times U C_{s}\right)\right\}$. Each element of the allocation, $r(n p, s, u c)$, denotes the part of the demand for service $s$, corresponding to the users of class $u c$, that should be satisfied by network provider $n p \quad\left(n p \in N P_{\text {avail }}\right)$. Network provider $n p$ should satisfy the service demand portion at the selected quality level $q l(s, u c)$. It holds that $r(n p, s, u c) \leq$ $d(s, u c)$.

The objective function that should be optimised by the allocations is denoted as $O F\left(A_{Q L}, A_{N P}\right)$, and is associated with the utility measures and the costs achieved by the allocations. Our target is to maximise this objective function, which can be expressed as:

$$
\begin{aligned}
& O F\left(A_{Q L}, A_{N P}\right)= \\
& \sum_{s \in S} \sum_{u c \in U C_{s}} d(s, u c) \cdot u_{s, u c, q l(s, u c)}-\sum_{s \in S} \sum_{u c \in U C_{s}} \sum_{n p \in N P_{\text {avail }}} r(s, u c, n p) \cdot c(n p, s, q l(s, u c))
\end{aligned}
$$

The allocations (selected quality levels and network providers) should respect some sets of constraints. The first one imposes the satisfaction of the service demand pattern and it can be expressed as:

$$
\sum_{n p \in N P_{\text {avail }}} r(n p, s, u c)=d(s, u c), \forall(s, u c) \in\left(S \times U C_{s}\right)
$$

The second set of constraints guarantees that the capacity limitations of the selected NPs will not be violated. In this point, we introduce the function $L\left(n p, A_{Q L}, A_{N P}\right)$, which represents the load that will be imposed on network provider $n p\left(n p \in N P_{\text {avail }}\right)$ as a result of the allocations $A_{Q L}$ and $A_{N P}$. So:

$$
L\left(n p, A_{Q L}, A_{N P}\right) \leq L_{\max }(n p) \forall n p \in N P_{\text {avail }}
$$

Another set of constraints should guarantee that the tariffs that will be imposed to the users should be compliant with the specifications in their profiles. This can be expressed as

$$
\begin{gathered}
c(n p, s, q l(s, u c))<\bar{c}_{s, u c, q_{s}(u c)}, \forall(s, u c) \in\left(S \times U C_{s}\right), \\
\forall n p \in N P_{\text {avail }}:(r(n p, s, u c)>0)
\end{gathered}
$$


Here, it should be noted that the solution of allocation of the service demand to quality levels and network providers will be forwarded to the SP-SCC- $i$, SP-GPC and the service plane mechanisms of the chosen NPs. The SP-SCC- $i$ will use this new solution for continuing the work at the session configuration layer. The SP-GPC will update its global view of the manner, in which users are served and services are provided in the service area. The whole problem, as it was described in this section, may have the graphical presentation of figure 2 .

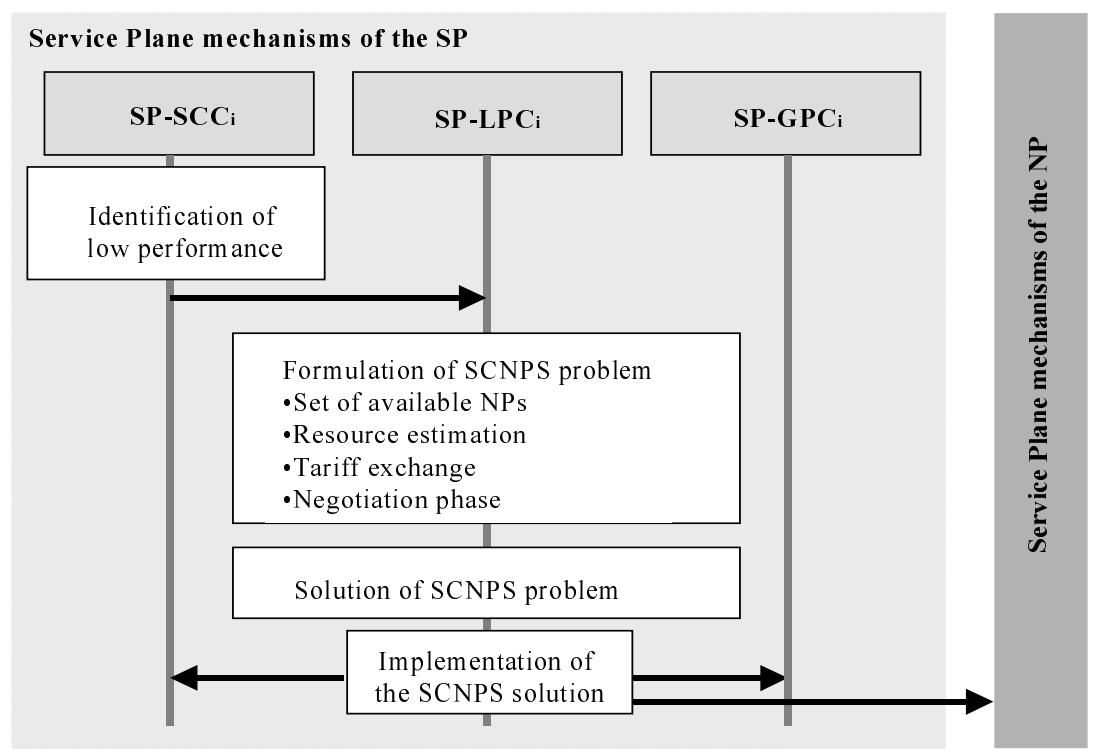

Fig. 2. Description of the Local Planning Layer functionality

\section{Results}

In this section, we intend to present a set of results for the evaluation of the proposed management techniques. More specifically, we analyse the problem of NP selection for the support of services in a future wireless cellular system, addressing two major possible solutions, the degradation in the quality of offered services and the binding of resources from alternative network providers. These solutions are evaluated according to the objective function, as it was described in the previous section. This function simulates the behaviour of the SP, according to the policies of the available NPs, as well as it takes into consideration the users' preferences, as they have been stored in their profiles. Finally, we evaluate the results using the concept of channel utilisation, which can be defined as $\mathrm{CU}=(1-\mathrm{P})^{*} \mathrm{~A} / \mathrm{n}$, where $P$ is the blocking probability, $A$ is the offered load and $n$ is the number of channels. 
For the purposes of this paper, we assume that the SP can choose between two NPs, $n p_{1}$ and $n p_{2}$ respectively, in order to engage resources for the support of a specific kind of service, which is not appropriately served within a portion of the service area. This service can be related to two quality levels, denoted as $Q L_{1}$ and $Q L_{2}$ respectively, both of them corresponding to $1 \%$ blocking probability. During the subscription of a user to a certain user class of the SP, the SP becomes aware of the user's profile and it can, therefore, estimate a utility measure for the allocation of the user to a specific quality level. Table 1 summarizes some typical values for the input parameters of the SCNPS problem.

Table 1. SP and NP policies for the SCNPS problem

\begin{tabular}{||c||c|c|c|c|c|}
\hline \multicolumn{1}{|c||}{} & \multicolumn{2}{c|}{ Utility Measure } & \multicolumn{2}{c|}{ Cost /Tariff } & \multirow{2}{*}{$\begin{array}{c}\text { Bit Rate } \\
\text { (Kbps) }\end{array}$} \\
\cline { 2 - 5 } & $U C_{l}$ & $U C_{2}$ & $N P_{1}$ & $N P_{2}$ & 384 \\
\hline$Q L_{l}$ & 5 & - & 1.5 & 3.5 & 144 \\
\hline$Q L_{2}$ & 5 & 2.5 & 0.75 & 1.75 & 144 \\
\hline
\end{tabular}

It should be noted here that we take into consideration the structure of a CDMA cellular system, with typical values of the channel bandwidth $\Delta f=5 \mathrm{MHz}$ and chip rate $C R=4.096 M c p s$. We, also, make the assumption that the SP has initially bound 16 channels from the cheapest NP. All users, who have already entered the system, have been accommodated to $n p_{1}$, which appears to be cheaper than $n p_{2}$, and they have been allocated at the high quality level $Q L_{1}$. In an instance of time, the offered load in the area, which is approximately OfferedLoad $=12$ Erlang and can be shared equally to both of classes, that is $\operatorname{Tr}_{U C_{1}}=6$ Erlang and $T r_{U C_{2}}=6$ Erlang, performs a $5 \%$ blocking probability, which means that the $S P-S C C_{i}$ has to request for a modification in configuration. There are two possible solutions:

- $n p_{1}$ has no more resources to allocate, so the SP determines to degrade the quality in low priority users. In this case, the new traffic in the area will be modified to OfferedLoad ${ }_{N E W}=8.5$ Erlang . Then, the objective function value is: $O F=31.5$.

- The SP can buy resources from $n p_{2}$. Our target is to keep the blocking probability at $B=1 \%$. For this reason, we need to transfer an amount of traffic from the low priority class UC2 to $n p_{2}$, in order to keep all of them in the high quality level. From Erlang B table, we can see that for 16 channels and $B=1 \%$ the offered load in $n p_{1}$ must be approximately 9 Erlang, from which an amount of 6 Erlang refers to the traffic of $U C_{1}$ and 3 Erlang to the traffic of $U C_{2}$. The rest 3 Erlang 
of the traffic of $U C_{2}$ will be served from $n p_{2}$. Thus, the objective function will become: $O F=36$.

We now examine the effect of the tariff policy of $n p_{2}$ in the objective function. On figure 3, we have declared 5 schemes of different tariff policies for $n p_{2}$ and the respective objective function for the case that the SP buys resources from that NP. On the same figure, we have depicted the objective function for the case of degrading the quality in low priority users, which remains as is for all schemes, since the alterations in the tariff policy of $n p_{2}$ have no effect on that case.

As it is illustrated from this figure, the solution of buying resources from an alternative NP (SP-SMS approach), keeping all the connections in the high quality level, is superior than the other one, that implies the degradation of the low priority connections, in order to keep all of them in the same NP. Only if the difference between the cost policies of the various available NPs exceeds a threshold, the proposed solution is not profitable. This threshold implies that the case of degradation the low priority users is preferable from SP's perspective, when $n p_{2}$ becomes at about $350 \%$ more expensive than $n p_{1}$. However, it would be safe to say that this threshold seems difficult to be reached, unless in very scarce circumstances.

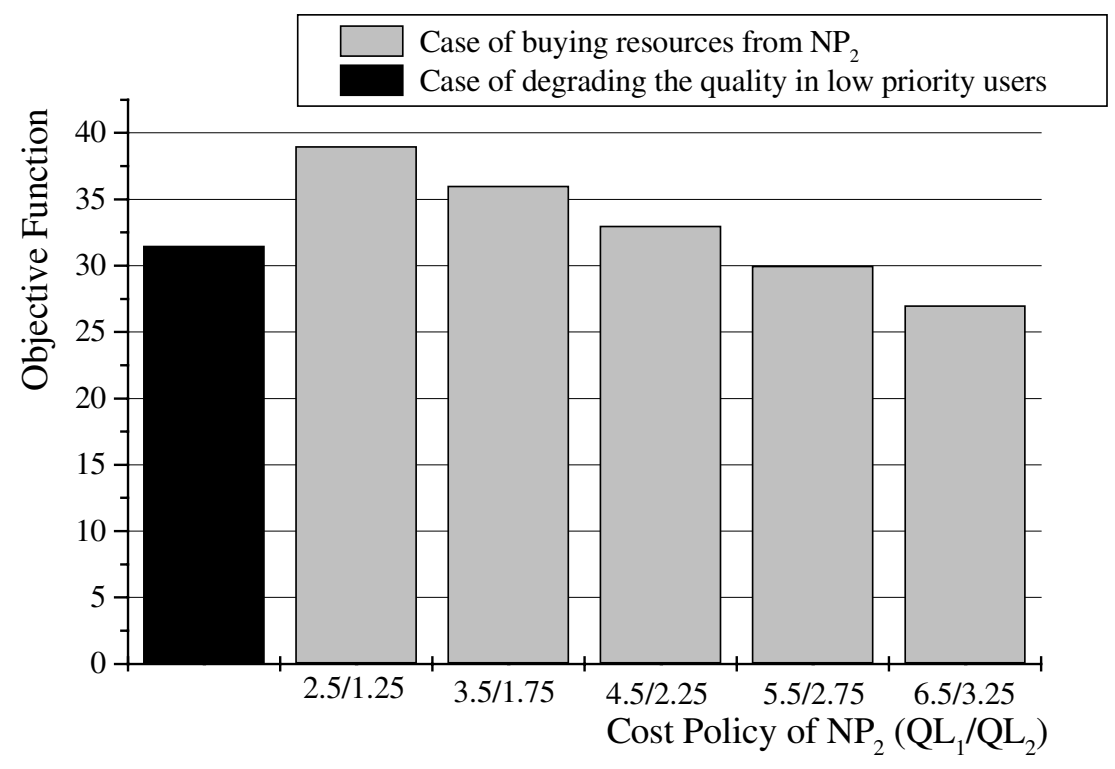

Fig. 3. The objective function vs. the cost policy of $n p_{2}$

We, now, examine the effect of utility measure on the SCNPS problem. Specifically, we assume that the SP's revenue by allocating $U C_{1}$ to $Q L_{1}$ and $Q L_{2}$ is 
5 and 0 units respectively. We also assume that the cost values for $n p_{1}$ are $c\left(n p_{1}, Q L_{1}\right)=1.5$ and $c\left(n p_{1}, Q L_{2}\right)=0.75$ and for $n p_{2}$ are $c\left(n p_{2}, Q L_{1}\right)=3$ and $c\left(n p_{2}, Q L_{2}\right)=1.5$. This means that we have assumed a reasonable scenario for the tariff policies of the available NPs, which implies a double cost policy of $n p_{2}$ regarding $n p_{1}$. Figure 4 depicts the objective function for 4 scenarios of the utility measure and for the allocation of $U C_{2}$ to respective quality levels. From this figure, we can conclude that, when the utility measure of user classes for quality levels is high, the SP-SMS leads to better performance. As the utility measure decreases, the SP-SMS logic gives worse results than the degradation case, when the user has no strong interest for the quality levels.

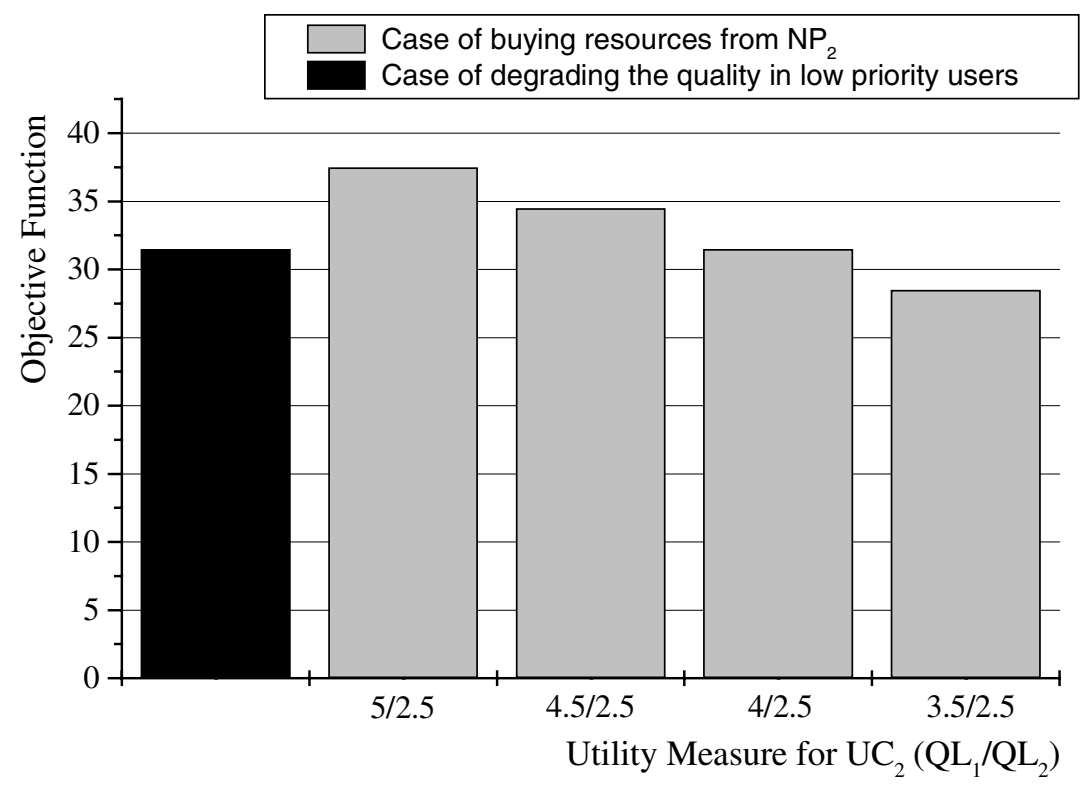

Fig. 4. The objective function vs. the utility measure of $U C_{2}$

From NPs' perspective, we examine the impact of the above scenario in the channel utilisation. The results can be found on table 2. From it, we can conclude that the capability of choice between different NPs for the support of a set of services in a specific area is much more preferable than the degradation in the quality of the offered services that some users experience. The channel utilisation is better for each NP separately, as well as the system as a whole. 
Table 2. Channel Utilisation for the cases under study

\begin{tabular}{|c|c|c|}
\hline \multirow{2}{*}{} & \multicolumn{2}{|c|}{ Channel Utilisation } \\
\cline { 2 - 3 } & $N P_{1}$ & $N P_{2}$ \\
\hline Case 1 & 0.495 & 0 \\
\hline Case 2 & 0.549 & 0.330 \\
\hline
\end{tabular}

\section{Conclusions}

The main feature of the future wireless cellular systems is the intelligence and flexibility in the provision of services. In this respect, we presented in this paper a Service Management System, from the SP's perspective, which has been classified into three layers and enables the SP to co-operate with a set of available NPs, in order to find the most appropriate one, according to cost-effective and user-satisfaction criteria.

The SP-SMS deals with the incoming sessions and allocates users' requests to different quality levels, according to the availability of resources. In case of low performance within a service area portion, there is an invocation for modification in its configuration. The objective of the management system is to find the best allocation of the service demands to quality levels and available NPs. The solution of the SCNPS problem must be communicated to the rest of SP-SMS layers and the service plane mechanisms of the associated NP.

\section{References}

1. S. Trigila, A. Mullery, M. Campolargo, J. Hunt, "Service architectures and service creation for integrated broadband communications", Computer Communications, Vol. 18, No. 11,1995

2. S. Trigila, K. Raatikainen, B. Wind, P. Reynolds, "Mobility in long-term service architectures and distributed platforms", IEEE Personal Commun., Vol. 5 No. 4, Aug. 1998

3. "Management models for telecommunications", Feature topic in the IEEE Commun. Mag., March 1996

4. V.Garg, D.Ness-Cohn, T.Powers, L.Schenkel, "Direction for element managers and network managers", IEEE Commun. Mag., October 1998

5. IST project MONASIDRE (Management of networks and services in a diversified radio environment) Web site, www.monasidre.com. Feb. 2001

6. IST project SHUFFLE (An agent based approach to controlling resources in UMTS) Web site, www.ist-shuffle.org. Jan. 2001

7. J.-T.Park, J.-W.Baek, J.W.-K.Hong, "Management of service level agreements for multimedia Internet service using a utility model”, IEEE Commun. Mag., Vol. 39, No. 5, May 2001

8. U. Varshney, "Recent advances in wireless networking", IEEE Computer, Vol. 33, No. 6, June 2000

9. A.K.Talukdar, B.R.Badrinath, A.Acharya, "Rate adaptation schemes in networks with mobile hosts", Proc. ACM/IEEE MobiCom'98, pp. 169-180, Oct.1998 
$910 \quad$ V. Tountopoulos et al.

10. Sunghyun Choi and Kang G. Shin, "Location/Mobility-Dependent Bandwidth Adaptation in QoS-Sensitive Cellular Networks," Proc. IEEE Vehicular Technology Conference, October 2001

11. "Design of broadband multiservice networks", Feature topic in the IEEE Commun. Mag., Vo. 36, No. 5, May 1998 